\title{
Osteoarthritis of the Knee: Assessing the Disease
}

\section{Przemyslaw Tomasz Paradowski ${ }^{1,2 *}$}

${ }^{1}$ Department of Orthopedics and Traumatology, Medical University, Clinical Hospital WAM, Żeromskiego 113, PL-90-549 Łódź, Poland

${ }^{2}$ Sunderby Central Hospital of Norrbotten, Sjukhusvägen 10, SE-971 80 Luleå, Sweden

\begin{abstract}
Knee osteoarthritis (OA) is the most common joint disease. It affects about $12 \%$ of adults 60 years of age but its prevalence is expected to rise. The incidence of OA increases with age but the disease can be stable for a long time and could reactivate some years later. Diagnosis of OA is primarily based on history and physical examination and on radiographic findings, including joint space narrowing (JSN), osteophyte formation and subchondral sclerosis. The radiographic examination of involved joints is also useful in assessing the severity of the disease. However, considerable discrepancy may exist between symptoms and the radiographic findings. The review gives proposed approaches to define symptomatic, progressive and end-stage knee OA. It focuses on the available scoring methods and their various advantages and disadvantages.
\end{abstract}

Keywords: Knee osteoarthritis; Radiographic progression; Magnetic resonance imaging; Knee outcomes

\section{Introduction}

Osteoarthritis (OA) is the most frequent musculoskeletal joint disorder. It is a major cause of pain and disability and the most common indication of joint replacement surgery. Though knee OA is most prevalent in general population, hip OA and hand OA are equally disabling, especially in older people. For the purpose of epidemiologic investigation, OA can be defined as a pathological process, development of radiographic changes and last, but not least, as a sequence of clinical symptoms.

\section{Prevalence and Incidence of $\mathrm{OA}$}

Knee OA appeared to be the primary diagnosis of 430,000 hospital discharges yearly in the United States [1]. The disease is strongly agerelated but gender-specific differences are also evident. In persons younger than 50 years of age, the prevalence of OA is higher in men but in older subjects women become much more frequently affected [2]. The community-based analyses showed that both the prevalence and incidence of OA increased 2 to 10 -fold between 30 and 65 years of age and continued to rise even later [3]. It has been estimated that the prevalence of knee OA is $12 \%$ in adults 60 years of age or older [4]. The number of people affected with OA is likely to increase. This phenomenon is undoubtedly related both to the aging of population and growing rate of overweight and obesity. $\mathrm{OA}$ is predicted to reach $30 \%$ of population older than 60 years of age by the year 2030 [5]. The annual incidence rates of knee OA range from $2 \%$ to $4 \%[6,7]$.

\section{Pathogenesis}

OA is a disease with multifactorial provenience. All subjects who have developed OA are probably affected by both genetics and environmental factors with individually distributed predominance. It has previously been reported that the risk of post-traumatic OA after a meniscal injury of the knee is strongly affected by a family history of $\mathrm{OA}$, by the presence of generalized OA (like the nodal osteoarthritis of the hand), by obesity, and by sex [8-10]. Many subjects with OA in one joint have the disease at different stages in other joints. The condition is usually expressed as a mixture of different features including inflammation, osteophyte formation and cartilage loss. However, since several of the environmental risk factors such as obesity, joint injury and joint overload are of biomechanical nature, OA is primarily a mechanical problem.
Joint changes result usually from either abnormal mechanical strains that affect healthy cartilage or from failure of pathologically impaired cartilage that degenerates under the influence of physiological mechanical strains. In the cases with mechanical origin, the pathology is focal and not generalized.

In general, the disease occurs when the dynamic equilibrium between the destruction and repair of joint tissues is impaired. The degenerative process starts at joint cartilage level and is characterized by a discrepancy between cartilage production and degradation. The process is, however, not limited to cartilage alone but is considered as a chronic disease of all joint tissues including subchondral bone, menisci, ligaments, and per articular muscles [11]. It consequently leads to joint instability and muscle weakness [12-14] that nowadays can be regarded as early features of structural and functional joint failure [15].

In the natural course of $\mathrm{OA}$, one can distinguish the symptomatic phase, the radiographic stage and the end-stage that demands knee replacement surgery.

\section{Clinical Manifestation}

When the joint failure begins, it is hard to say. At the beginning the condition can be asymptomatic, but the later the structural joint failure is characterized with disabling pain and joint dysfuction. Clinical assessment of patients with joint symptoms allows evaluating limitation in the range of movement, deformities and instability.

For assessment of joint function, symptoms and disability the disease-specific and patient-related outcomes (PROs) have been developed. In OA, the Index of Severity for osteoarthritis of the Knee (ISK) [16], Western Ontario and McMaster Universities osteoarthritis index (WOMAC) [17] and the Knee injury and Osteoarthritis Outcome

*Corresponding author: Przemyslaw Tomasz Paradowski, Sunderby Central Hospital of Norrbotten, Sjukhusvägen 10, SE-971 80 Luleå, Sweden, Tel: + 46708329574 ; E-mail: przemyslaw.t.paradowski@gmail.com

Received February 14, 2014; Accepted February 18, 2014; Published February 20, 2014

Citation: Paradowski PT (2014) Osteoarthritis of the Knee: Assessing the Disease. Health Care Current Reviews 2: e103. doi: 10.4172/2375-4273.1000e103

Copyright: (c) 2014 Paradowski PT. This is an open-access article distributed under the terms of the Creative Commons Attribution License, which permits unrestricted use, distribution, and reproduction in any medium, provided the original author and source are credited. 
Score (KOOS) $[18,19]$ belong to those used most widely. PROs are not sufficient for diagnosis but very suitable to follow the progression of symptoms in clinical trials and for long-term assessment of effectiveness of therapeutic interventions [20-22].

\section{Radiographic knee OA}

When OA is advanced, its features are visible on plain radiographs which show narrowing of joint space (due to cartilage loss), osteophytes, and sometimes changes in the subchondral bone. Confirmation of radiographic changes with $\mathrm{X}$-ray has long been considered as the reference standard.

The most common method for radiographic evaluation of OA is the osteophyte-based Kellgren-Lawrence (K/L) joint scoring system that grades joint changes in five levels from 0 to 4 (where 0 corresponds to a healthy joint) [23]. K/L grading defines OA by the presence of a definite osteophyte (corresponding to grade $\geq 2$ ) with minor focus on joint space narrowing, bone sclerosis, cysts, and joint deformity [2,23]. However, the interpretation was with time considered inadequate. Since there was no consistency if grade 2 should be defined in terms of narrowing of the joint space, the same patients could be defined as having and not having OA [24-26]. Thus, scales that could more accurately describe the disease were introduced. The Ahlbäck classification focuses primarily on the reduction of the joint space as an indirect sign of cartilage loss [27]. Indeed, joint space narrowing has been suggested as the best variable in assessing radiographic progression of knee OA $[11,28]$. The Ahlbäck grading system has not, however, gained a widespread popularity and is used mainly in northern Europe [29,30].

In order to investigate the incidence and progression of $\mathrm{OA}$ in epidemiologic studies and clinical trials of disease modifying therapies, a yet more sensitive classification was needed. The Osteoarthritis Research Society International (OARSI) atlas is now considered to be the best approach to describe the OA in different joints. The scale is based on semi-quantitative examination of individual radiographic features, such as osteophytes and joint space narrowing [31,32].

\section{Symptomatic OA}

Symptomatic OA is generally defined by the presence of pain, aching or stiffness in a joint with radiographic OA. Symptomatic OA is classically defined as a combination/coincidence of radiographic tibiofemoral OA corresponding to Kellgren-Lawrence scale grade of $\geq 2$ (at least mild radiographic OA) and symptoms in the same knee. Syptomatic OA appears to be common but it rarely progresses to its severe form. Only part of subjects with OA seeks medical help and in these cases the symptomatic treatment is usually sufficient. Symptomatic OA has however been regarded as a primary indication for most of knee replacements [33].

\section{Progression of $\mathrm{OA}$}

OA has generally been regarded as a progressive disorder. However, it is not always so. When evaluating larger population groups, it has been observed that the progression rate is small but that a fast progression can occur in some individuals $[21,34,35]$. On a subject level, OA can be stable for a long time and could reactivate some years later. It is consistent with the fact that only a small proportion of people in the community with OA ultimately need joint replacement [36]. Large epidemiological investigations as the Framingham Study or the Rotterdam Study that used K/L scale have defined new-onset disease as the emergence of $\mathrm{K} / \mathrm{L}$ grade 2 in the knee joints that had previously been graded 0 or 1 . New-onset of incident radiographic OA has usually been defined as the development of osteophyte, and thus assessment ignored the progression of joint space narrowing, manifestation of cartilage loss.

Radiographic knee OA progression could also be evaluated by the change in the radiographic scores calculated by adding JSN and osteophytes grades in medial and lateral knee compartments. Radiographic progression can be defined as an increase in score by at least 1 (which we considered as representing the smallest detectable change, unpublished data).

\section{End-stage OA}

In some patients, OA reaches with time the state in which the surgical intervention such as osteotomy or total knee replacement should be scheduled. There is, however, no general consensus how much developed the disease must be to be assessed as the end stage $\mathrm{OA}$ in subjects who have not been operated on. OA can be considered to reach its end-stage if JSN and osteophytes' grade was 3 according to OARSI atlas [31,32].

Radiographic examination of the knee joint is regarded as a standard assessment that allows describing the joint changes and thus diagnosing $\mathrm{OA}$. It has been reported that the reproducibility in radiographic joint space measurement is weak. Then, a systemic measurement error can emerge especially when the knee is examined in extended position due to variations in knee pain between the examinations and affected ability to extend the joint.

Plain radiography is useful for defining OA, but has weak associations with symptoms, limited sensitivity to change, as well as poor prediction of cartilage loss and the need for joint replacement $[37,38]$. Since joint degeneration exists before the $\mathrm{x}$-ray is able to show radiographic changes, the value of radiographic examination, especially in early phases of OA is nowadays debatable. It would be recommended to find a method defining the disease in a different way.

\section{Magnetic Resonance Imaging}

Contrary to radiographic examination in which JSN is considered to be a surrogate marker for cartilage damage [39], magnetic resonance imaging (MRI) allows to assess cartilage more directly [40]. MRI has the capability to visualize multiple joint structures and is especially useful to detect early cartilage changes and subchondral bone marrow abnormalities (that has been shown to be predictors of radiographic progression) [41,42]. It has been found that there is a correlation between progression of JSN on radiographic images and cartilage loss in MRI [43]. However, the substantial proportion of knees with cartilage loss could be missed when analyzed only on standard X-rays.

The use of MRI is still limited, partly due to its costs and partly due to technical demands. The assessments need standardized procedures and methods to interpret results. Examination of patients could be sometimes difficult, eg. because of the subject's obesity or feeling of claustrophobia. It is also difficult to compare results obtained from different systems, which is relevant for longitudinal multicenter investigations [40].

\section{Knee Arthroscopy}

Although arthroscopy has no relevance as a treatment method and therefore is not indicated in subjects with knee OA, it is often performed when the knee injury (such as meniscus tear) is suspected in individuals with no priorly diagnosed OA. The discussion if the subjects with symptoms typical for OA with concomitant changes 
seen during knee arthroscopy could be defined as having OA is ongoing. Indeed, arthroscopy allows assessing only changes within the internal joint structures and therefore does not seem to be suitable to describe the whole picture of the disease. The correlation between arthroscopic Outerbridge classification [44,45] (which describes only cartilage condition but says nothing about osteophytes and joint space narrowing) was recorded to be poor, especially in women with milder cartilage affection [46].

\section{Conclusion}

$\mathrm{OA}$ of the knee joint is already a problem which is expected to get more and more severe as we can observe a rise in knee injuries and obesity in population. Although much progress in understanding the disease has been done in the past several decades, we still do not know enough to assess the risk of joint failure, to prevent it and last but not least manage it effectively in its early phase. It seems to be necessary to be able to define OA or joint failure as early as possible with no need to wait for confirmation that disease has reached its developed stage. Future research is needed to find and standardize the methods of joint evaluation that could select patients with knee pain that might suggest a pre-OA stage, which, in turn, could help us treat them more effectively.

\section{References}

1. Agency for Healthcare Research and Quality (2006) National and regional statistics in the national inpatient sample, 2004.

2. Zhang Y, Jordan JM (2010) Epidemiology of Osteoarthritis. Clin Geriatr Med 26: 355-369

3. Oliveria SA, Felson DT, Reed JI, Cirillo PA, Walker AM (1995) Incidence of symptomatic hand, hip, and knee osteoarthritis among patients in a health maintenance organization. Arthritis Rheum 38: 1134-1141.

4. Dillon CF, Rasch EK, Gu Q, Hirsch R (2006) Prevalence of knee osteoarthritis in the United States: arthritis data from the Third National Health and Nutrition Examination Survey 1991-94. J Rheumatol 33: 2271-2279.

5. Croft $P$ (2005) The epidemiology of osteoarthritis: Manchester and beyond. Rheumatology (Oxford) 44: 27-32

6. Felson DT, Zhang Y, Hannan MT, Naimark A, Weissman BN, et al. (1995) The incidence and natural history of knee osteoarthritis in the elderly. The Framingham Osteoarthritis Study. .Arthritis Rheum 38: 1500-1505.

7. Hart DJ, Doyle DV, Spector TD (1999) Incidence and risk factors for radiographic knee osteoarthritis in middle-aged women: the Chingford Study. Arthritis Rheum 42: 17-24.

8. Doherty M, Watt I, Dieppe P (1983) Influence of primary generalised osteoarthritis on development of secondary osteoarthritis. Lancet 8340: 8-11.

9. Englund M, Paradowski P, Lohmander LS (2004) Radiographic hand osteoarthritis is associated with radiographic knee osteoarthritis after meniscectomy. Arthritis Rheum 50: 469-475.

10. Englund M, Lohmander LS (2004) Risk factors for symptomatic knee osteoarthritis fifteen to twenty-two years after meniscectomy. Arthritis Rheum 50: 2811-2819.

11. Hart DJ, Spector TD (1995) The classification and assessment of osteoarthritis. Baillières Clin Rheumatol 9: 407-432.

12. Hurley MV (1999) The role of muscle weakness in the pathogenesis of osteoarthritis. Rheum Dis Clin N Amer 25: 283-298.

13. Sharma L (2003) Examination of exercise effects on knee osteoarthritis outcomes: why should the local mechanical environment be considered? Arthritis Rheum 49: 255-260.

14. Sharma $L$ (2003) Local mechanical factors in the natural history of knee osteoarthritis. Malalignment and joint laxity. (2ndedn). Oxford University Press, Oxford.

15. Nuki G (1999) Osteoarthritis: a problem of joint failure. Z Rheumatol 58: 142 147.
16. Lequesne MG, Mery C, Samson M, Gerard $P$ (1987) Indexes of severity of osteoarthritis of the hip and knee. Validation-Value in comparison with other assessment tests. Scand J Rheumatol 65: 85-89.

17. Bellamy N, Buchanan WW, Goldsmith CH, Campbell J, Stitt LW (1988) Validation study of WOMAC: a health status instrument for measuring clinically important patient relevant outcomes to antirheumatic drug therapy in patients with osteoarthritis of the hip or knee. J Rheumatol 15: 1833-1840.

18. Roos EM, Roos HP, Ekdahl C, Lohmander LS (1998) Knee injury and Osteoarthritis Outcome Score (KOOS)--validation of a Swedish version. Scand J Med Sci Sports 8: 439-448.

19. Roos EM, Roos HP, Lohmander LS, Ekdahl C, Beynnon BD (1998) Knee Injury and Osteoarthritis Outcome Score (KOOS) - development of a selfadministered outcome measure. J Orthop Sports Phys Ther 28: 88-96.

20. Roos EM, Lohmander LS (2003) The Knee injury and Osteoarthritis Outcome Score (KOOS): from joint injury to osteoarthritis. Health Qual Life Outcomes 1: 64 .

21. Paradowski PT, Englund M, Roos EM, Lohmander LS (2004) Similar group mean scores, but large individual variations, in patient-relevant outcomes over 2 years in meniscectomized subjects with and without radiographic knee osteoarthritis. Health Qual Life Outcomes 2: 38.

22. Paradowski PT, Englund M, Lohmander LS, Roos EM (2005) The effect of patient characteristics on variability in pain and function over two years in early knee osteoarthritis. Health Qual Life Outcomes 3: 59.

23. Kellgren J, Lawrence J (1957) Radiological assessment of osteoarthritis. Ann Rheum Dis 16: 494-502.

24. Schiphof D, Boers M, Bierma-Zeinstra SM (2008) Differences in descriptions of Kellgren and Lawrence grades of knee osteoarthritis. Ann Rheum Dis 67: 1034-1036.

25. Schiphof D, de Klerk BM, Kerkhof HJ, Hofman A, Koes BW, et al. (2011) Impact of different descriptions of the Kellgren and Lawrence classifi cation criteria on the diagnosis of knee osteoarthritis. Ann Rheum Dis 70: 1422-1427.

26. Felson DT, Niu J, Guermazi A, Sack B, Aliabadi P (2011) Defining radiographic incidence and progression of knee osteoarthritis: suggested modifications of the Kellgren and Lawrence scale. Ann Rheum Dis 70: 1884-1886.

27. Ahlbäck S (1968) Osteoarthrosis of the knee: a radiographic investigation. Acta Radiol Stockholm 277 : 7-72

28. Altman RD, Fries JF, Bloch DA, Carstens U, Cooke TD, et al. (1987) Radiographic assessment of progression in osteoarthritis. Arthritis Rheum 30: 1214-1225.

29. Bergström G, Bjelle A, Sundh V, Svanborg A (1986) Joint disorders at ages 70 75, and 79 years. A cross-sectional comparison. Br J Rheumatol 25: 333-341.

30. Pettersson IF, Boegård T, Saxne T, Silman AJ, Svensson B (1997) Radiographic osteoarthritis of the knee classified by the Ahlbäck and Kellgren \& Lawrence systems for the tibiofemoral joint in people aged 35-54 years with chronic knee pain. Ann Rheum Dis 56: 493-496.

31. Altman RD, Hochberg M, Murphy Jr WA, Wolfe F, Lequesne M (1995) Atlas of individual radiographic features in osteoarthritis. Osteoarthritis Cartilage 3 : 3-70.

32. Altman RD, Gold GE (2007) Atlas of individual radiographic features in osteoarthritis, revised. Osteoarthritis Cartilage 15: A1-56.

33. Katz JN, Barrett J, Mahomed NN, Baron JA, Wright RJ, et al. (2004) Association between hospital and surgeon procedure volume and the outcomes of total knee replacement. J Bone Joint Surg Am 86: 1909-1916.

34. Dieppe PA, Cushnaghan J, Shepstone L (1997) The Bristol 'OA500' study: progression of osteoarthritis (OA) over 3 years and the relationship between clinical and radiographic changes at the knee joint. Osteoarthritis Cartilage 5 : 87-97.

35. Dieppe P, Cushnaghan J, Tucker M, Browning S, Shepstone L (2000) The Bristol 'OA500 study': progression and impact of the disease after 8 years. Osteoarthritis Cartilage 8: 63-68.

36. Dieppe PA, Lohmander LS (2005) Pathogenesis and management of pain in osteoarthritis. Lancet 365: 965-973.

37. Cicuttini FM, Jones G, Forbes A, Wluka AE (2004) Rate of cartilage loss at two years predicts subsequent total knee arthroplasty: a prospective study. Ann Rheum Dis 63: 1124-1127. 
38. Cooper C, Snow S, McAlindon TE, Kellingray S, Stuart B, et al. (2000) Risk factors for the incidence and progression of radiographic knee osteoarthritis. Arthritis Rheum 43: 995-1000.

39. Hellio Le Graverand M-P, Mazzuca S, Duryea J, Brett A (2009) Radiographicbased grading methods and radiographic measurement of joint space width in osteoarthritis. Radiol Clin N Am 47: 567-579.

40. Guermazi A, Roemer FW, Burstein D, Hayashi D (2011) Why radiography should no longer be considered a surrogate outcome measure for longitudinal assessment of cartilage in knee osteoarthritis. Arthritis Res Ther 13: 247.

41. Koster IM, Oei EH, Hensen JH, Boks SS, Koes BW, et al. (2011) Predictive factors for new onset or progression of knee osteoarthritis one year after trauma: MRI follow-up in general practice. Eur Radiol 21: 1509-1516.
42. Ding C, Cicuttini F, Jones G (2008) How important is MRI for detecting early osteoarthritis? Nature Clinical Practice Rheumatology 4: 4-5.

43. Amin S, LaVally MP, Guermazi A, Grigorian M, Hunter DJ, et al. (2005) The relationship between the cartilage loss on magnetic resonance imaging and radiographic progression in men and women with knee osteoarthritis. Arthritis Rheum 52: 3152-3159.

44. Outerbridge RE (1961) The etiology of chondromalacia patellae. J Bone Joint Surg $\mathrm{Br}$ 43: 752-757.

45. Outerbridge RE (1964) Further studies on the etiology of chondromalacia patellae. J Bone Joint Surg Br 46: 179-190.

46. Al Omran AS (2009) Osteoarthritis of knee: correlation between radiographic and arthroscopic findings. Int Surg 94: 269-272. 